Siberian Mathematical Journal, Vol. 57, No. 2, pp. 322-329, 2016

Original Russian Text Copyright (c) 2016 Kytmanov A.A., Osipov N.N., and Tikhomirov S.A.

\title{
FINDING EIN COMPONENTS IN THE MODULI SPACES OF STABLE RANK 2 BUNDLES ON THE PROJECTIVE 3-SPACE
}

(c) A. A. Kytmanov, N. N. Osipov, and S. A. Tikhomirov

UDC 512.7

\begin{abstract}
Some method is proposed for finding Ein components in moduli spaces of stable rank 2 vector bundles with first Chern class $c_{1}=0$ on the projective 3 -space. We formulate and illustrate a conjecture on the growth rate of the number of Ein components in dependence on the numbers of the second Chern class. We present a method for calculating the spectra of the above bundles, a recurrent formula, and an explicit formula for computing the number of the spectra of these bundles.
\end{abstract}

DOI: $10.1134 /$ S0037446616020142

Keywords: stable bundle, Chern class, moduli space

\section{Introduction}

We consider the spaces $M_{\mathbb{P}^{3}}(2 ; 0, n)$ of the moduli of stable rank 2 bundles with first Chern class $c_{1}=0$ and second Chern class $c_{2}=n$ on $\mathbb{P}^{3}$. The article focuses primary on the aspects of the number-theoretic study of the so-called Ein components - some special class of components in the spaces $M_{\mathbb{P}^{3}}(2 ; 0, n)$. We obtain all Ein components and find their exact number in the moduli space for any number of $c_{2}$. A condition is established for the existence of Ein components in an arbitrary (a certain) moduli space. In particular for $1 \leq c_{2} \leq 10^{6}$ the complete list of the numbers of the second Chern class for which the moduli spaces do not include Ein components is given. Moreover, we formulate and illustrate a conjecture on the growth rate of the number of Ein components as the second Chern class of the corresponding bundles grows. We also present a method for calculating all spectra of rank 2 bundles with $c_{1}=0$ on $\mathbb{P}^{3}$ satisfying necessary properties. The discovered Fibonacci-type recurrent relation makes it possible to obtain an exact formula for the number of these spectra. As an example, we consider the only Ein component in the moduli space of rank 2 bundles with $c_{1}=0$ and $c_{2}=14$ on $\mathbb{P}^{3}$, compute its dimension, and establish the correspondence of this component to a specific spectrum of bundles. We work over an algebraically closed field $\mathbf{k}$ of characteristic 0 .

\section{Available Results on the Components of $M_{\mathbb{P}^{3}}(2 ; 0, n)$}

The components of $M_{\mathbb{P}^{3}}(2 ; 0, n)$ are known for $1 \leq n \leq 4$ : for $n=1,2$, there is a unique component (see [1]), for $n=3,4$, there are two components (see [2,3]). For each $n \geq 1$, we know at least one component $I_{n}$ of $M_{\mathbb{P} 3}(2 ; 0, n)$, the so-called instanton component with expected dimension $\operatorname{dim} I_{n}=8 n-3$. The irreducibility of $I_{n}$ was proved by A. S. Tikhomirov for odd $n$ in [4] and then for even $n$ in [5]. The smoothness of $I_{n}$ was showed in [6].

Three other series of irreducible families (possibly, components) in $M_{\mathbb{P}^{3}}(2 ; 0, n)$ with $n$ ranging over an infinite sequence (but not with arbitrary $n$ ) were constructed by R. Hartshorne in [7] and V. Vedernikov in $[8]$.

The authors were supported by the Russian Foundation for Basic Research (Grant 15-31-20008-mol_a_ved, the first and third authors; Grant 14-01-00283-a, the first author); the Ministry of Education and Science of the Russian Federation (Government Task 1.1462.2014/K, the first and second authors), and the basic part of Government Task 1.14.365 (the third author).

Krasnoyarsk; Yaroslavl'; Koryazhma. Translated from Sibirskiて Matematicheskiน Zhurnal, Vol. 57, No. 2, pp. 410-419, March-April, 2016; DOI: 10.17377/smzh.2016.57.214. Original article submitted May 5, 2015. 
In [9], L. Ein considered a special class of stable vector bundles of rank 2 on $\mathbb{P}^{3}$, the class of the so-called generalized null correlation bundles $E_{2}$, which are cohomological sheaves of monads of the form

$$
0 \rightarrow \mathscr{O}_{\mathbb{P}^{3}}(-c) \rightarrow \mathscr{O}_{\mathbb{P}^{3}}(-b) \oplus \mathscr{O}_{\mathbb{P}^{3}}(-a) \oplus \mathscr{O}_{\mathbb{P}^{3}}(a) \oplus \mathscr{O}_{\mathbb{P}^{3}}(b) \rightarrow \mathscr{O}_{\mathbb{P}^{3}}(c) \rightarrow 0,
$$

where $c>b \geq a \geq 0$.

In this case, it is easy to calculate that $c_{1}\left(E_{2}\right)=0$ and $c_{2}\left(E_{2}\right)=c^{2}-a^{2}-b^{2}$. Moreover, Ein showed that these bundles are stable if and only if $c>a+b$ and that the moduli space $M_{\mathbb{P}^{3}}\left(2 ; 0, c^{2}-a^{2}-b^{2}\right)$ has an irreducible component $N(a, b, c)$ whose generic point corresponds to the class of bundles that are cohomological sheaves of monads (1). We refer to such components as Ein components.

Thus, the problem of finding the exact number of Ein components in the moduli space of rank 2 bundles with first Chern class $c_{1}=0$ is reduced, depending on the number $c_{2}=n$ of the second Chern class of $E_{2}$, to the problem of finding for given $n \geq 1$ all triples $(a, b, c)$ of integers satisfying the conditions

$$
c^{2}-a^{2}-b^{2}=n, \quad 0 \leq a \leq b, \quad c>a+b .
$$

This problem is addressed in Section 2 .

The study of these components is closely connected with the notion of "spectrum" of the bundle. In the case of an arbitrary characteristic, the notion of the spectrum for a stable bundle of rank 2 with $c_{1}=0$ on $\mathbb{P}^{3}$ was introduced by Hartshorne [7]. Namely, let Spec $E_{2}$ be the spectrum of a rank 2 bundle with $c_{1}=0$. Then Spec $E_{2}=\left(a_{1}, a_{2}, \ldots, a_{n}\right)$, where $a_{1}, a_{2}, \ldots, a_{n}$ is a nondecreasing sequence of integers possessing the following properties (see $[7,10])$ :

(S1) symmetry: $\left(a_{n}, a_{n-1}, \ldots, a_{1}\right)=-\left(a_{1}, a_{2}, \ldots, a_{n}\right)$;

(S2) connectedness: for every two numbers in $\operatorname{Spec} E_{2}$, each number between them also belongs to $\operatorname{Spec} E_{2}$;

(S3) if a number $l_{0}$ such that $1 \leq l_{0} \leq \max \left\{a_{i}\right\}$ appears in the spectrum Spec $E_{2}$ only once then each number $l$ such that $l_{0} \leq l \leq \max \left\{a_{i}\right\}$ must also appear in Spec $E_{2}$ only once.

For example, the bundles $E_{2}$ with lexicographically minimal spectrum, the zero spectrum $(0, \ldots, 0)$, are exactly the instanton bundles which are open dense subsets in the instanton components $I_{n}$.

All possible numbers for the spectra of rank 2 bundles with $c_{1}=0$ and the forms of the monads whose cohomological sheaves are these bundles are listed in [10] for $1 \leq c_{2} \leq 8$.

In Section 4 we consider the problem of finding the spectra of stable rank 2 bundles with $c_{1}=0$ on $\mathbb{P}^{3}$.

\section{A Method for Finding Ein Components and Its Justification}

Given a positive integer $n$, denote by $T_{n}$ the number of triples $(a, b, c)$ of nonnegative integers satisfying the system

$$
c^{2}-a^{2}-b^{2}=n, \quad c>a+b .
$$

Refer to a triple $(a, b, c)$ as suitable if $a \leq b$. Denote the number of suitable triples by $t_{n}$. Also let $P_{n}$ be the number of suitable triples with $a=b$. Clearly,

$$
t_{n}=\frac{T_{n}+P_{n}}{2}
$$

Let us describe an algorithm that allows us to list all suitable triples $(a, b, c)$ for a positive integer $n$. Replacing $c$ by $k=c-a-b$, we get

$$
(a+k)(b+k)=\frac{n+k^{2}}{2},
$$

where $k \geq 1$ and $k \equiv n(\bmod 2)$. Since $0 \leq a \leq b$, we also have

$$
\frac{n+k^{2}}{2} \geq(a+k)^{2} \geq k^{2}
$$


Consequently, we come to the constraints

$$
k \equiv n \quad(\bmod 2), \quad 1 \leq k \leq \sqrt{n}, \quad 0 \leq a \leq \sqrt{\frac{n+k^{2}}{2}}-k .
$$

The number of pairs $(k, a)$ of integers satisfying (3) asymptotically equals

$$
\frac{1}{2} \int_{1}^{\sqrt{n}}\left(\sqrt{\frac{n+k^{2}}{2}}-k\right) d k \sim \frac{\sqrt{2} \log (1+\sqrt{2})}{8} n \approx 0.156 n .
$$

We enumerate all such pairs $(k, a)$ and for each of them check the condition

$$
n+k^{2} \equiv 0 \quad(\bmod 2(a+k)) .
$$

If, for some pair $(k, a)$, this condition is fulfilled then, putting

$$
b=\frac{n+k^{2}}{2(a+k)}-k, \quad c=a+b+k,
$$

we get a suitable triple $(a, b, c)$. In particular, the number $t_{n}$ of suitable triples $(a, b, c)$ is equal to the number of pairs $(k, a)$ satisfying (3) and (4).

Now, for a given positive integer $n$, consider the problem of finding all suitable triples $(a, b, c)$ with $a=b$. The corresponding algorithm is similar. We must find all pairs $(k, a)$ of integers $k \geq 1$, $a \geq 0$, for which

$$
\frac{n+k^{2}}{2}=(a+k)^{2} \text {. }
$$

Each such pair $(k, a)$ gives a suitable triple of the form $(a, a, 2 a+k)$. All desired pairs $(k, a)$ can be listed starting from the constraints

$$
k \equiv n \quad(\bmod 2), \quad 1 \leq k \leq \sqrt{n}, \quad a=\sqrt{\frac{n+k^{2}}{2}}-k .
$$

For estimating the number $P_{n}$ of such pairs, write down the last equation in the form

$$
2(a+k)^{2}-k^{2}=n \text {. }
$$

To each integer solution $(k, a)$ to this equation with $k \geq 1, a \geq 0$, there corresponds a solution $(x, y)=$ $(k, a+k)$ to the norm equation

$$
\left|x^{2}-2 y^{2}\right|=n
$$

satisfying the inequalities $1 \leq x \leq y$. It is not hard to check that any two such solutions $(x, y)$ cannot be associated; therefore,

$$
P_{n} \leq Q(n),
$$

where $Q(n)$ is the maximal number of pairwise nonassociated solutions to (5). We have

$$
Q(n)=\sum_{d \mid n} \chi(d)
$$

where $\chi$ is a character of the quadratic field $\mathbb{Q}(\sqrt{2})$; i.e.,

$$
\chi(d)=\left\{\begin{aligned}
+1, & d \equiv 1,7(\bmod 8) \\
-1, & d \equiv 3,5(\bmod 8) \\
0, & \text { otherwise }
\end{aligned}\right.
$$

(see [11, Chapter III, $\S 8$, Problem 17]). In particular, $q_{n}^{*} \leq \tau(n)$, where $\tau(n)$ is the number of divisors of $n$.

The computer experiments imply that

$$
t_{n}^{\mathrm{av}}=\frac{t_{1}+\cdots+t_{n}}{n} \sim \operatorname{const} \cdot n^{1 / 2}
$$

where const $\approx 0.2617$. Indeed, we have 
Proposition 1. The asymptotics

$$
t_{n}^{\mathrm{av}}=\frac{t_{1}+\cdots+t_{n}}{n} \sim \frac{\pi}{12} \cdot n^{1 / 2}
$$

holds.

Proof. Indeed, firstly,

$$
\begin{gathered}
T_{n}^{\mathrm{av}}=\frac{T_{1}+\cdots+T_{n}}{n} \sim \frac{1}{n} \int_{0}^{\infty} d a \int_{0}^{(2 a)^{-1} n}\left(\sqrt{a^{2}+b^{2}+n}-a-b\right) d b \\
=n^{1 / 2} \int_{0}^{\infty} d \xi \int_{0}^{(2 \xi)^{-1}}\left(\sqrt{\xi^{2}+\eta^{2}+1}-\xi-\eta\right) d \eta=n^{1 / 2} \int_{0}^{\infty} \frac{\left(1+\xi^{2}\right) \log \left(1+\xi^{-2}\right)-1}{4} d \xi=\frac{\pi}{6} n^{1 / 2}
\end{gathered}
$$

since $T_{1}+\cdots+T_{n}$ is the number of triples $(a, b, c)$ of nonnegative integers satisfying the system

$$
1 \leq c^{2}-a^{2}-b^{2} \leq n, \quad c>a+b .
$$

Secondly,

$$
P_{n}^{\mathrm{av}}(n)=\frac{P_{1}+\cdots+P_{n}}{n} \sim \frac{1}{n} \int_{1}^{\sqrt{n}}\left(\sqrt{\frac{n+k^{2}}{2}}-k\right) d k \sim \frac{\sqrt{2} \log (1+\sqrt{2})}{4} \approx 0.312
$$

since $P_{1}+\cdots+P_{n}$ is the number of pairs $(k, a)$ of integers $k \geq 1$ and $a \geq 0$ satisfying the inequalities $1 \leq 2(a+k)^{2}-k^{2} \leq n$. Applying (2) completes the proof of the statement.

Note also that $Q(1)+\cdots+Q(n)$ is the number of pairs $(x, y)$ of integers for which

$$
\left|x^{2}-2 y^{2}\right| \leq n, \quad 1 \leq x+y \sqrt{2}<1+\sqrt{2} .
$$

Consequently,

$$
Q^{\mathrm{av}}(n)=\frac{Q_{1}+\cdots+Q_{n}}{n} \sim \frac{1}{2 \sqrt{2}} \int_{1}^{1+\sqrt{2}} \frac{d \xi}{\xi}=\frac{\sqrt{2} \log (1+\sqrt{2})}{2} \approx 0.623 .
$$

Thus, (6) is twice higher in the mean.

\section{Asymptotic Behavior of the Numbers of Ein Components}

The number-theoretic result by Ein (see [9, Lemma 3.5]) asserts that if the second Chern class $c_{2}=n$ of a rank 2 bundle with $c_{1}=0$ is a product of $m$ different odd numbers then the number of suitable triples $(a, b, c)$ (respectively, the number of Ein components) is at least $m$. As is seen from (7) and (8), this estimate is rather rough in the mean. For example, for $n=315=5 \cdot 7 \cdot 9$, Ein's result gives a lower bound for the number of triples equal to 3 , whereas $t_{315}=12$. As a consequence, Ein proves the assertion that the number $t_{n}$ of all different irreducible components of $M_{\mathbb{P}^{3}}(2 ; 0, n)$ satisfies the equality $\varlimsup_{n \rightarrow \infty} t_{n}=\infty$. The computer experiment, presented in the article, allows us to make a more accurate conjecture on the growth rate of $t_{n}$.

The algorithm for computing all suitable triples $(a, b, c)$ is implemented in the Maple computer algebra system. Using the latter, we obtained experimental data on the number of triples for the numbers of the 
second Chern class from 1 to $10^{6}$, which allows us to formulate the following conjecture:

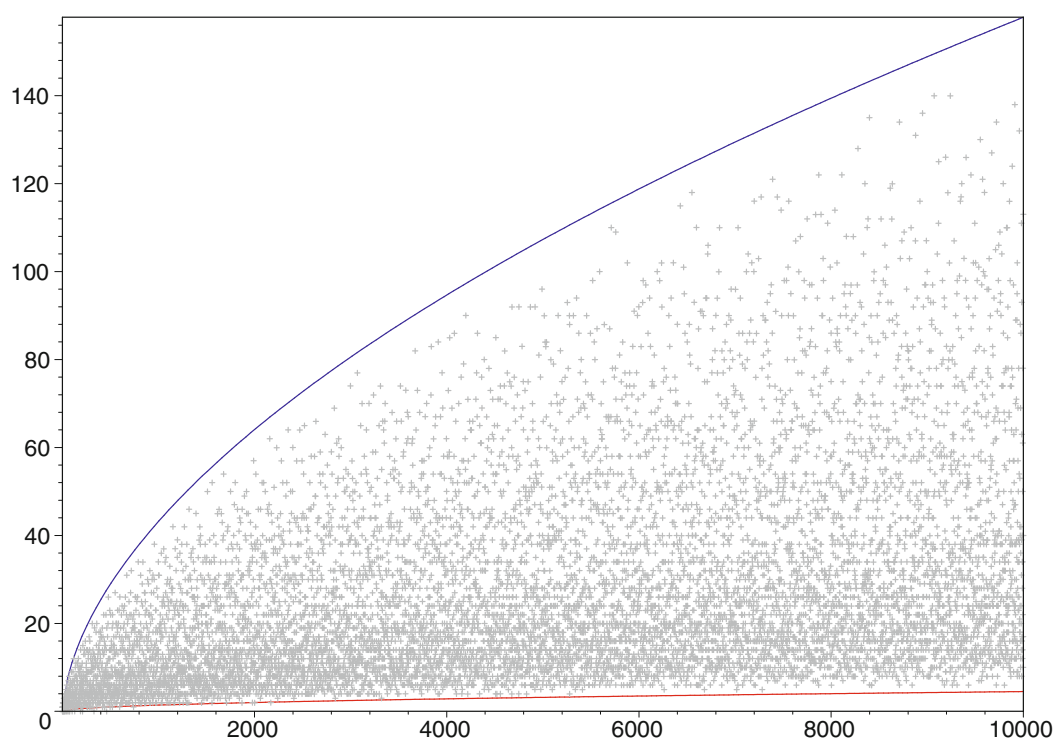

Fig. 1. The numbers $t_{n}$ of suitable triples (Ein components)

Conjecture 1. Let $N$ be a positive integer number and let $n$ be the number of the second Chern class of a bundle $E_{2}$ with zero first Chern class. Then

$$
0.045 \sqrt{n} \leq t_{n} \leq 0.52 \sqrt{n \log n}
$$

for all $n>N$, where $t_{n}$ is the number of suitable triples (Ein components).

This conjecture is illustrated in Fig. 1, where the numbers $t_{n}$ of suitable triples are marked by dots for $n=1, \ldots, 10^{4}$, and the upper and lower functions are the graphs of the functions $0.52 \sqrt{x \log x}$ and $0.045 \sqrt{x}$ respectively. It is worth noting that to obtain some exact bounds like (9) for $t_{n}$ is a rather challenging number-theoretic problem. The following remark is about the asymptotic behavior of $t_{n}$ 's "from below."

REMARK 1. The definitions of $t_{n}$ and $T_{n}$ imply the equivalence of the inequalities $t_{n}>0$ and $T_{n}>0$. In turn, the computer experiments show that, in case $n \leq 10^{6}$, we have $T_{n}=0$ only for $n \in\{2,6,10,18,22,30,42,58,70,78,102,130,190,210,330,462\}$. It is not hard to see that the possible exclusions can be only among the numbers of the form $p-1$, where $p$ is a prime with $p \equiv 3(\bmod 4)$.

\section{Finding the Spectra of Stable Rank 2 Bundles}

As Hartshorne observed in [7], the spectra of bundles carry out stratification of the moduli spaces of the bundles, which facilitates the study of the spaces. In particular, information about the (attainable) spectra aids in studying the "geography" and geometry of the components in moduli spaces, making it possible to split the problem of studying a certain moduli space (its components) into a smaller problem.

For the convenience of subsequent arguments, we introduce

Definition 1. Refer as a spectrum-vector of length $k$ to an integer-valued vector $\left(v_{1}, \ldots, v_{k}\right)$ of length $k$ with nonnegative elements such that

(1) $v_{1}=0$

(2) $v_{i+1}=v_{i}$ or $v_{i+1}=v_{i}+1$ for all $i \in\{1, \ldots$,$\} ;$

(3) if $v_{i-1}<v_{i}<v_{i+1}$ for some $i \in\{2, \ldots, k-1\}$ then $v_{j}<v_{j+1}$ for all $j \in\{i+1, \ldots, k-1\}$.

Conditions (2) and (3) in Definition 1 coincide with the corresponding properties (S2) and (S3) of the spectrum of a rank 2 bundle with first Chern class $c_{1}=0$. 
Note that there is a one-to-one correspondence between a spectrum-vector $\left(0, v_{2}, \ldots, v_{k}\right)$ of length $k$ and a spectrum of length $n$, namely, for odd $n=2 k-1$, the corresponding spectrum has the form $\left(-v_{k}, \ldots,-v_{2}, 0, v_{2}, \ldots, v_{k}\right)$, and for even $n=2 k$, the form $\left(-v_{k}, \ldots,-v_{2}, 0,0, v_{2}, \ldots, v_{k}\right)$.

It is easy to verify that the only spectrum-vector of length 1 is (0), and the spectrum-vectors of length 2 are $(0,0)$ and $(0,1)$. The spectrum-vectors of length at least 3 satisfy

Theorem 1. For $m=1,2, \ldots$, each of the spectrum-vectors of length $m+2$ is one of the following vectors:

(1) a spectrum-vector of length $m+1$ with zero added on the left;

(2) the sum of a spectrum-vector of length $m$ with two zeros added on the left and the vector $(0,1, \ldots, 1)$ of length $m+2$;

(3) the vector $(0,1,2, \ldots, m+1)$.

ProOF. By property (3) of the definition of spectrum-vector, for $m=1,2, \ldots$, each spectrum-vector of length $m+2$ belongs to one of the following types:

(1) the vectors of the form $(0,0, \ldots)$ which we will call vectors of the first type;

(2) the vectors of the form $(0,1,1, \ldots)$ which we will call vectors of the second type;

(3) the vector $(0,1,2, \ldots, m+1)$.

Each vector of the first type is a spectrum-vector of length $m+1$ with zero added on the right. Indeed, observe that any spectrum-vector starts with 0 , and fixing one more element 0 in fact diminishes the dimension of the vector by 1 . Thus, we obtain all spectrum-vectors of length $m+1$.

Now, consider a spectrum-vector of the second type. Note that all its elements but the first are at least 1 ; therefore, its difference with $(0,1, \ldots, 1)$ is a nonnegative integer-valued vector of the form $(0,0,0, \ldots)$. Subtraction of this vector does not influence the conditions (2) and (3) of the definition of spectrum. Therefore, three fixed zeros at the beginning diminish the dimension of the vector by 2 , and we obtain the set of the spectrum-vectors of length $m$ with two zeros added on the left.

By property (3) of the definition of spectrum-vector, the only spectrum-vector of length $m+2$ that is not a vector of the first or second type is $(0,1,2, \ldots, m+1)$.

The algorithm for computing spectra of stable rank 2 bundles based on the above result was implemented in the Maple computer algebra system.

Corollary 1. For $k=1,2, \ldots$, the sequence of the numbers $a_{k}$ of spectrum-vectors of length $k$ satisfies the recurrent relation $a_{k+2}=a_{k+1}+a_{k}+1$ with initial data $a_{1}=1, a_{2}=2$.

It is easy to see that $a_{k}=F_{k+2}-1$, where $F_{k}$ is the $k$ th Fibonacci number,

$$
F_{k}=\frac{(1+\sqrt{5})^{k}-(1-\sqrt{5})^{k}}{2^{k} \sqrt{5}} .
$$

Thus, we obtained an explicit formula for calculating the number of spectrum-vectors of length $k$ (and hence of the number of spectra with properties (S1)-(S3)).

REMARK 2. It was shown in [10] that the spectra of rank 2 bundles with $c_{1}=0$ and $1 \leq c_{2} \leq 19$ with properties (S1)-(S3) are actually attainable; i.e., there exist rank 2 bundles having such spectra, and there are no other attainable spectra. Respectively, for bundles with $c_{1}=0$ and $c_{2} \geq 20$, the numbers $a_{k}$ are upper bounds for the numbers of attainable spectra.

\section{An Example of Finding the Dimension of an Ein Component}

By way of example, consider the Ein components of $M_{\mathbb{P}^{3}}(2 ; 0,14)$ in more detail. We have

Theorem 2. $M_{\mathbb{P}^{3}}(2 ; 0,14)$ has the unique Ein component: the component of dimension 117 containing the dense open subset of the classes of bundles with spectrum $(-3,-2,-2,-1,-1,-1,0,0,1,1,1$, $2,2,3)$ and defined by the monad of the type

$$
0 \rightarrow \mathscr{O}_{\mathbb{P}^{3}}(-4) \rightarrow 2 \mathscr{O}_{\mathbb{P}^{3}}(-1) \oplus 2 \mathscr{O}_{\mathbb{P}^{3}}(1) \rightarrow \mathscr{O}_{\mathbb{P}^{3}}(4) \rightarrow 0 .
$$


Proof. Using the algorithm of Section 2 we find the unique suitable triple, where $(a, b, c)=(1,1,4)$. Thus, $M_{\mathbb{P} 3}(2 ; 0,14)$ has exactly one Ein component.

Let us find the spectrum of rank 2 bundles $E_{2}$ in this Ein component and calculate its dimension.

In our case, monad $(1)$ has the form $0 \rightarrow \mathscr{O}_{\mathbb{P}^{3}}(-4) \rightarrow 2 \mathscr{O}_{\mathbb{P}^{3}}(-1) \oplus 2 \mathscr{O}_{\mathbb{P}^{3}}(1) \rightarrow \mathscr{O}_{\mathbb{P}^{3}}(4) \rightarrow 0$. Hence, the display of $(11)$, tensored by $\mathscr{O}_{\mathbb{P}^{3}}(-1)$, is as follows:

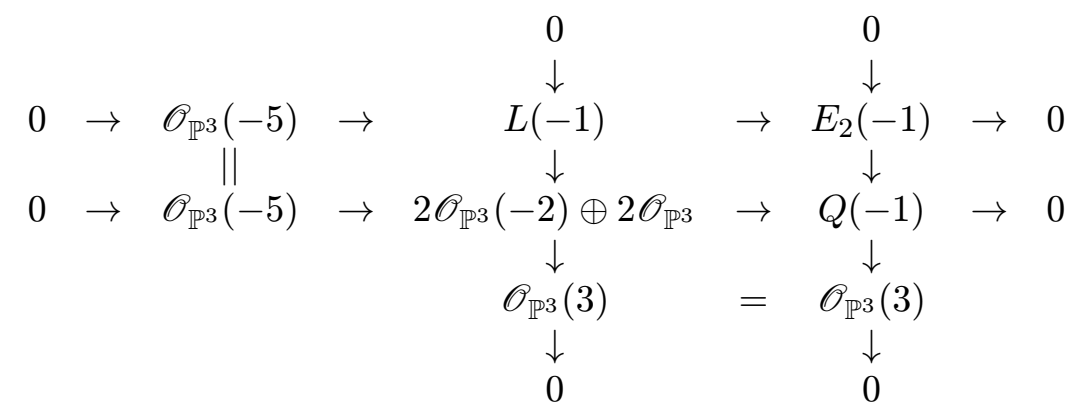

where $L$ and $Q$ are some sheaves. Using this display and taking into account the stability of $E_{2}$, we find

$$
h^{1} E_{2}(-1)=18 .
$$

Similarly, considering the displays for $E_{2}(-2-i), i \geq 0$, we obtain

$$
h^{1} E_{2}(-2)=10, h^{1} E_{2}(-3)=4, h^{1} E_{2}(-4)=1, h^{1} E_{2}(-5-i)=0, i \geq 0 .
$$

The result of Section 4 makes it possible to obtain the exact number of the attainable spectra for stable rank 2 bundles with $c_{1}=0$ and $c_{2}=14$ equal to 33 . List these spectra:

$$
\begin{aligned}
& \text { \#1. }(0,0,0,0,0,0,0,0,0,0,0,0,0,0) \text {; } \\
& \text { \#3. (-1, -1, 0, 0, 0, 0, 0, 0, 0, 0, 0, 0, 1, 1); } \\
& \text { \#5. (-1, -1, -1, 0, 0, 0, 0, 0, 0, 0, 0, 1, 1, 1); } \\
& \text { \#7. (-3, -2, -1, 0, 0, 0, 0, 0, 0, 0, 0, 1, 2,3); } \\
& \text { \#9. }(-2,-1,-1,-1,0,0,0,0,0,0,1,1,1,2) \text {; } \\
& \text { \#11. (-3, -2, -1, -1, 0, 0, 0, 0, 0, 0, 1, 1, 2,3); } \\
& \text { \#13. (-1, -1, -1, -1, -1, 0, 0, 0, 0, 1, 1, 1, 1, 1); } \\
& \text { \#15. (-2, -2, -1, -1, -1, 0, 0, 0, 0, 1, 1, 1, 2, 2); } \\
& \text { \#17. (-2, - 2, - 2, -1, - 1, 0, 0, 0, 0, 1, 1, 2, 2, 2); } \\
& \text { \#19. (-4, -3, - 2, -1, -1, 0, 0, 0, 0, 1, 1, 2, 3, 4); } \\
& \text { \#21. (-1, -1, -1, -1, -1, -1, 0, 0, 1, 1, 1, 1, 1, 1); } \\
& \text { \#23. (-2, - 2, -1, -1, -1, -1, 0, 0, 1, 1, 1, 1,2,2); } \\
& \text { \#25. (-2, - 2, -2, -1, -1, -1, 0, 0, 1, 1, 1, 2, 2, 2); } \\
& \text { \#27. (-4, -3, -2, -1, -1, -1, 0, 0, 1, 1, 1, 2,3,4); } \\
& \text { \#29. (-3, -2, -2, -2, -1, -1, 0, 0, 1, 1, 2, 2, 2, 3); } \\
& \text { \#31. (-4, -3, -2, -2, -1, -1, 0, 0, 1, 1, 2, 2, 3, 4); } \\
& \text { \#33. (-6, -5, -4, -3, -2, -1, 0, 0, 1, 2, 3, 4, 5, 6). }
\end{aligned}
$$$$
\text { \#2. (-1,0,0,0,0,0,0,0,0,0,0,0,0,1); }
$$$$
\text { \#4. }(-2,-1,0,0,0,0,0,0,0,0,0,0,1,2) \text {; }
$$$$
\text { \#6. }(-2,-1,-1,0,0,0,0,0,0,0,0,1,1,2) \text {; }
$$$$
\text { \#8. }(-1,-1,-1,-1,0,0,0,0,0,0,1,1,1,1) \text {; }
$$$$
\text { \#10. }(-2,-2,-1,-1,0,0,0,0,0,0,1,1,2,2) \text {; }
$$$$
\text { \#12. (-4, -3, -2, -1, 0, 0, 0, 0, 0, 0, 1, 2,3,4); }
$$$$
\text { \#14. (-2, -1, -1, -1, -1, 0, 0, 0, 0, 1, 1, 1, 1,2); }
$$$$
\text { \#16. (-3, - 2, -1, -1, -1, 0, 0, 0, 0, 1, 1, 1, 2,3); }
$$$$
\text { \#18. (-3, - 2, -2, -1, -1, 0, 0, 0, 0, 1, 1,2,2,3); }
$$$$
\text { \#20. (-5, -4, -3, - 2, -1, 0, 0, 0, 0, 1, 2, 3, 4, 5); }
$$$$
\text { \#22. (-2, -1, -1, -1, -1, -1, 0, 0, 1, 1, 1, 1, 1,2); }
$$$$
\text { \#24. (-3, - 2, -1, -1, -1, -1, 0, 0, 1, 1, 1, 1, 2,3); }
$$$$
\text { \#26. (-3, -2, - 2, -1, -1, -1, 0, 0, 1, 1, 1, 2, 2,3); }
$$$$
\text { \#28. (-2, -2, -2, -2, -1, -1, 0, 0, 1, 1, 2, 2, 2, 2); }
$$$$
\text { \#30. (-3, -3, -2, -2, -1, -1, 0, 0, 1, 1, 2, 2,3,3); }
$$$$
\text { \#32. (-5, -4, -3, -2, -1, -1, 0, 0, 1, 1, 2, 3, 4, 5); }
$$

Consider spectrum \#26 in this list. Using Barth's techniques (see [12]), we have $h^{1} E_{2}(-1)=h^{0} K$, $h^{1} E_{2}(-2)=h^{0} K(-1), h^{1} E_{2}(-3)=h^{0} K(-2)$, and $h^{1} E_{2}(-4)=h^{0} K(-3)$, where $K=\oplus_{k} \mathscr{O}_{\mathbb{P}^{1}}(k)$, and the numbers $k$ range over the above spectra. From this, by elementary calculations, we infer that equalities (10) and (11) hold for the spectrum Spec $E_{2}=(-3,-2,-2,-1,-1,-1,0,0,1,1,1,2,2,3)$, which is listed under number \#26. By the uniqueness of the spectrum Spec $E_{2}$ of $E_{2}$, it follows that the Ein component corresponds to this spectrum. Applying Barth's formula (see [12]), we find the dimension $d$ of the Ein component:

$$
d=d_{1}-d_{2}-d_{3}-d_{4}
$$


where

$$
\begin{gathered}
d_{1}=\operatorname{dim} \operatorname{Hom}\left(2 \mathscr{O}_{\mathbb{P}^{3}}(-1) \oplus 2 \mathscr{O}_{\mathbb{P}^{3}}(1),\right. \\
\left.\mathscr{O}_{\mathbb{P}^{3}}(4)\right)=2 h^{0} \mathscr{O}_{\mathbb{P}^{3}}(5)+2 h^{0} \mathscr{O}_{\mathbb{P}^{3}}(3)=112+40=152 ; \\
d_{2}=\operatorname{dim} \operatorname{Hom}\left(\mathscr{O}_{\mathbb{P}^{3}}(4), \mathscr{O}_{\mathbb{P}^{3}}(4)\right)=h^{0} \mathscr{O}_{\mathbb{P}^{3}}=1 ; \\
d_{3}=h^{0}\left(\Lambda^{2}\left(\mathscr{O}_{\mathbb{P}^{3}}(4)\right)\right)=0 ; \\
d_{4}=h^{0}\left(S^{2}\left(2 \mathscr{O}_{\mathbb{P}^{3}}(-1) \oplus 2 \mathscr{O}_{\mathbb{P}^{3}}(1)\right)\right)=h^{0}\left(S^{2}\left(2 \mathscr{O}_{\mathbb{P}^{3}}(-1)\right)\right)+h^{0}\left(S^{2}\left(2 \mathscr{O}_{\mathbb{P}^{3}}(1)\right)\right)+4 h^{0} \mathscr{O}_{\mathbb{P}^{3}} \\
=3 h^{0} \mathscr{O}_{\mathbb{P}^{3}}(-2)+3 h^{0} \mathscr{O}_{\mathbb{P}^{3}}(2)+4=0+30+4=34 .
\end{gathered}
$$

Thus, $d=152-1-0-34=117$, and we obtain a component of dimension greater than the expected one (which is equal to $8 \cdot 14-3=109$ in this case).

\section{References}

1. Hartshorne R., "Stable vector bundles of rank 2 on $\mathbb{P}_{3}$," Math. Ann., 238, 229-280 (1978).

2. Barth W., "Irreducibility of the space of mathematical instanton bundles with rank 2 and $c_{2}=4$," Math. Ann., Bd 258, Heft 1, 81-106 (1981).

3. Ellingsrud G. and Strömme S., "Stable rank 2 vector bundles on $\mathbb{P}^{3}$ with $c_{1}=0$ and $c_{2}=3$," Math. Ann., Bd 255, 129-138 (1981).

4. Tikhomirov A. S., "Moduli of mathematical instanton vector bundles with odd $c_{2}$ on projective space," Izv. Math., 76, No. 5, 991-1073 (2012).

5. Tikhomirov A. S., "Moduli of mathematical instanton vector bundles with even $c_{2}$ on projective space," Izv. Math., 77, No. 6, 1195-1223 (2013).

6. Jardim M. and Verbitsky M., "Trihyperkahler reduction and instanton bundles on $\mathbb{C} P^{3}$," Compos. Math., 150, No. 11, 1836-1868 (2014).

7. Hartshorne R., "Stable reflexive sheaves," Math. Ann., 254, 121-176 (1980).

8. Vedernikov V. K., "Moduli of stable vector bundles of rank 2 on $\mathbb{P}^{3}$ with fixed spectrum," Math. USSR-Izv., 25, No. 2, 301-313 (1985).

9. Ein L., "Generalized null correlation bundles," Nagoya Math. J., 111, 13-24 (1988).

10. Hartshorne R. and Rao A. P., "Spectra and monads of stable bundles," J. Math. Kyoto Univ., 31, No. 3, 789-806 (1991).

11. Borevich Z. I. and Shafarevich I. R., Number Theory, Acad. Press, New York (1966).

12. Barth W., "Some experimental data," Les Equations de Yang-Mills. Seminaire E.N.S. 1977-1978. Asterisque, 71-72. 1980. P. 205-218.

A. A. Kytmanov; N. N. Osipov

Siberian Federal University, Krasnoyarsk, Russia

E-mail address: aakytm@gmail.com; nnosipov@rambler.ru

S. A. TIKHOMIROV

Yaroslavl' State Pedagogical University, Yaroslavl', Russia

Koryazhma Branch of Northern (ARCtic) Federal University, Koryazhma, Russia

E-mail address: satikhomirov@mail.ru 